\title{
Long non-coding RNA SNHG17 promotes lung adenocarcinoma progression by targeting the microRNA-193a-5p/NETO2 axis
}

\author{
ZHIWEI ZHANG ${ }^{1,2}$, YULAN YAN $^{2,3}$, BIN ZHANG $^{1}$, YUCHEN MA $^{1}$, CHEN CHEN $^{1}$ and CHANGLI WANG ${ }^{1}$ \\ ${ }^{1}$ Department of Lung Cancer, Tianjin Medical University Cancer Institute and Hospital, \\ National Clinical Research Center for Cancer, Key Laboratory of Cancer Prevention and Therapy, \\ Tianjin's Clinical Research Center for Cancer, Tianjin 300060; Departments of ${ }^{2}$ Thoracic Surgery and \\ ${ }^{3}$ Teaching and Research, The Fifth Central Hospital of Tianjin, Tianjin 300450, P.R. China
}

Received March 18, 2021; Accepted August 4, 2021

DOI: $10.3892 / \mathrm{ol} .2021 .13079$

\begin{abstract}
Long non-coding RNAs (lncRNAs) play vital roles in human cancers. It has been reported that lncRNA SNHG17 expression is dysregulated in different types of cancer and involved in cancer progression. However, the role of SNHG17 in lung adenocarcinoma (LUAD) remains unclear. The present study aimed to investigate the role of SNHG17 in LUAD. Reverse transcription-quantitative (RT-q) PCR analysis was performed to detect SNHG17 expression in LUAD tissues and cells. The effects of SNHG17 on cancer cell migration, invasion, proliferation and epithelial-to-mesenchymal transition (EMT) were assessed via Transwell, MTT and western blot assays, respectively. The interactions between SNHG17 and microRNA (miRNA/miR)-193a-5p, miR-193a-5p and neuropilin and tolloid-like 2 (NETO2) were assessed via the dual-luciferase reporter assay. NETO2 expression and its potential role in LUAD were analyzed via RT-qPCR analysis and the UALCAN database. The results demonstrated that SNHG17 expression was significantly upregulated in LUAD tissues and cells, and high SNHG17 expression was associated with tumor-node-metastasis stage and poor prognosis of patients with LUAD. SNHG17 knockdown inhibited cell migration, invasion, proliferation and the EMT process. In addition, the results revealed that SNHG17 functions as a competing endogenous RNA of miR-193a-5p. The results of the dual-luciferase reporter assay confirmed that miR-193a-5p can directly target SNHG17. NETO2 was also predicted as a target protein of miR-193a-5p, which was confirmed via the
\end{abstract}

Correspondence to: Professor Changli Wang, Department of Lung Cancer, Tianjin Medical University Cancer Institute and Hospital, National Clinical Research Center for Cancer, Key Laboratory of Cancer Prevention and Therapy, Tianjin's Clinical Research Center for Cancer, 1 Huan-Hu-Xi Road, Ti-Yuan-Bei, He Xi, Tianjin 300060, P.R. China

E-mail: wangchangli@tjmuch.com

Key words: lung adenocarcinoma, SNHG17, microRNA-193a-5p, neuropilin and tolloid-like 2 dual-luciferase reporter assay. The roles of NETO2 knockdown in cancer cells were rescued following transfection with miR-193a-5p inhibitor or overexpression of SNHG17. Notably, high NETO2 expression was associated with poor prognosis of patients with LUAD. Bioinformatics analysis demonstrated that the promoter methylation level of NETO2 decreased in LUAD. Taken together, the results of the present study suggest that SNHG17 expression is upregulated in LUAD tissues and cells, and SNHG17 exerts tumor promoting effect by targeting the miR-193a-5p/NETO2 axis.

\section{Introduction}

Lung cancer is one of the most common malignancies and one of the leading causes of cancer-associated mortality worldwide $(1,2)$. According to the major histopathology features, lung cancer is divided into non-small cell lung cancer (NSCLC) and small cell lung cancer (SCLC) (3). NSCLC includes squamous cell carcinoma, adenocarcinoma and large cell carcinoma (4), and accounts for $>80 \%$ of all patients with lung cancer (5). Lung adenocarcinoma (LUAD) is the predominant subtype of NSCLC, accounting for $\sim 50 \%$ (6). Despite advancements in the diagnosis and treatment strategies for LUAD, the overall survival rate of patients with LUAD remains poor, and the 5 -year overall survival is only $15 \%(7,8)$. Thus, it is important to determine the underlying pathogenic mechanisms of LUAD.

Long non-coding RNAs (lncRNAs) play pivotal roles in various biological events $(9,10)$. The aberrant expression of lncRNAs is closely associated with the development and progression of diseases, including cancer (9). In different types of cancer, lncRNAs serve as oncogenes or tumor suppressors to regulate the development and progression of tumors, thus, IncRNAs also function as biomarkers for diagnosis and treatment of cancers $(11,12)$. For example, lncRNA-CDC6 functions as a competitive endogenous RNA to promote CDC6 expression by directly binding to microRNA (miRNA/miR)-215, which promotes breast cancer progression (13). In hepatocellular carcinoma (HCC), IncRNA uc.134 expression is significantly downregulated, and its overexpression inhibits the proliferation, invasion and metastasis of HCC cells by inhibiting ubiquitination of LATS1 and increasing YAP phosphorylation (14). In addition, IncRNA TBILA and 
AGAP2-AS1 expression levels are upregulated in patients with NSCLC, and thus may be potential diagnostic biomarkers for NSCLC (15). IncRNA SNHG17 has been reported to play a vital role in different types of cancer, including colorectal, pancreatic and breast cancers (16-18). However, the molecular basis of SNHG17 in LUAD remains unclear.

Recent studies have reported that IncRNAs play roles in diseases by acting as ceRNAs to directly sponge miRNAs to upregulate mRNAs expression. For example, lncRNA TTN-AS1 is negatively associated with miR-142-5p expression in LUAD, and accelerates LUAD progression by promoting cell migration, invasion and epithelial-to-mesenchymal transition (EMT) by regulating the miR-142-5p/CDK5 axis (19). Overexpression of miR-130a-5p inhibits NSCLC cell proliferation and increases apoptosis; however, these effects are rescued by overexpressing KTN1-AS1 (20). In addition, LINC01123 is highly expressed and positively associated with poor prognosis of patients with NSCLC and promotes c-Myc mRNA expression by inhibiting miR-199a-5p expression (21).

The aim of the present study was to investigate the potential mechanism and roles of the SNHG17/miR-193a-5p/NETO2 axis in LUAD.

\section{Materials and methods}

Cancer samples. A total of 50 pairs of LUAD tumor tissues and corresponding adjacent normal tissues (4-5 cm from tumor tissue) were collected from patients with LUAD at the Tianjin Medical University Cancer Institute and Hospital (Tianjin, China) between June 2016 and June 2019. The tissue samples were stored at $-80^{\circ} \mathrm{C}$. Among them, there were 32 males and 18 females (age, $<60(n=26), 52 \pm 8 ; \geq 60(n=24), 69 \pm 9$. No patients received radiotherapy and/or chemotherapy prior to surgery. The inclusion criteria were as follows: i) patients diagnosed with LUAD; ii) had no other diseases, and iii) provided informed consent. Patients not meeting these criteria were excluded. Patient characteristics are listed in Table I. The data regarding EGFR mutation status and PD-L1 expression was obtained from patient records. The present study was approved by the Ethics Committees of Tianjin Medical University Cancer Institute and Hospital (Tianjin, China; approval no. TJWZXYXEC) and written informed consent was provided by all patients prior to the study start.

Cell culture. The normal lung cell line, BEAS-2B and the cancer cell lines, A549, H1299, H1650, H1975 and CALU-3 were purchased from the American Type Culture Collection. All cells were maintained in RPMI-1640 medium supplemented with $10 \%$ fetal bovine serum (FBS) (both from Gibco; Thermo Fisher Scientific, Inc.). The 293T cells were purchased from Chinese Academy of Sciences and maintained in DMEM medium (Gibco; Thermo Fisher scientific, Inc.) supplemented with $10 \%$ FBS. All cells were maintained at $37^{\circ} \mathrm{C}$ with $5 \% \mathrm{CO}_{2}$.

Reverse transcription-quantitative (RT-q) PCR. Total RNA was extracted from tumor tissues or cells using TRIzol ${ }^{\circledR}$ (Invitrogen; Thermo Fisher Scientific, Inc.). Total RNA (1 $\mu \mathrm{g})$ was reverse transcribed into cDNA using the cDNA synthesis kit (Invitrogen; Thermo Fisher Scientific, Inc.). The following temperature protocol for RT was used: $85^{\circ} \mathrm{C}$ for $5 \mathrm{sec}, 37^{\circ} \mathrm{C}$ for $15 \mathrm{~min}$, then the samples were stored at $4^{\circ} \mathrm{C}$. qPCR was subsequently performed on a Real-Time PCR machine (Applied Biosystems; Thermo Fisher Scientific, Inc.) using the SYBRGreen RT-PCR reagents kit (Takara Biotechnology Co., Ltd.) according to the manufacturer's instructions. The following thermocycling conditions were used: Initial denaturation at $95^{\circ} \mathrm{C}$ for $5 \mathrm{~min}$, then $95^{\circ} \mathrm{C}$ for $5 \mathrm{sec}, 60^{\circ} \mathrm{C}$ for $30 \mathrm{sec}$ for 40 cycles. U6 was the internal control of miR-193a-5p, while GAPDH was the internal control for SNHG17 and NETO2. Relative expression level of gene was calculated using the $2^{-\Delta \Delta \mathrm{Cq}}$ method (22). The following primer sequences were used for qPCR: SNHG17 forward, 5'-GTTCCTGGG GCTTGGATGAT-3' and reverse, 5'-GATCTAAGGCTGAGA CCCACG-3'; miR-193a-5p forward, 5'-GATAGCCTGATG TTATCA-3' and reverse, 5'-GTCTGGTGAGTTGGCG-3'; NETO2 forward, 5'-AGATGGGCCATTTGGTTTCTC-3' and reverse, 5'-TGCTCGAAATCCCAGTCCTTC-3'; GAPDH forward, 5'-ACAACT TTGGTATCGTGGAAGG-3' and reverse, 5'-GCCATCACGCCACAGTTTC-3'; and U6 forward, 5'-CTCGCTTCGGCAGCACA-3' and reverse, 5'-AACGCT TCACGAATTTGCGT-3'.

Cell transfection. To obtain the SNHG17 overexpression vector, the full-length sequence of SNHG17 was cloned into the pcDNA3.1 vector (Invitrogen; Thermo Fisher Scientific, Inc.). The short hairpin RNAs (shRNAs) of SNHG17 and negative control shRNA (sh-NC), small inhibiting (si) RNA $\mathrm{NC}$ and siRNA targeting NETO2 (50 nM) were synthesized by Shanghai GenePharma Co., Ltd. The miR-193a-5p mimics (5'-UGGGUCUUUGCGGGCGAGAUGA-3') and miR-193a-5p inhibitor (5'-UCAUCUCGCCCGCAAAGACC CA-3') were designed and synthesized by Guangzhou RiboBio Co., Ltd. The sequence of the NC mimics is 5'-UCACAACCU CCUAGAAAGAGUAGA-3'. The sequence of the NC inhibitor is 5'-UCUACUCUUUCUAGGAGGUUGUGA-3'. The concentration of mimics and inhibitor was $50 \mathrm{nM}$. The concentration of shRNA was $0.5 \mu \mathrm{g}$. Transfection was performed at $37^{\circ} \mathrm{C}$ for $6 \mathrm{~h}$, then the medium was changed. Subsequent experiments were performed after $48 \mathrm{~h}$ of transfection. All transfections were performed using Lipofectamine ${ }^{\circledR} 3000$ (Invitrogen; Thermo Fisher Scientific, Inc.), according to the manufacturer's instructions. The following sequences were used: sh-NC, 5'-UCUCCGAACGUGUCACGUU-3'; sh-SNHG17\#1, 5'-GGA TTGTCAGCTGACCTCTGT-3' and sh-SNHG17\#2, 5'-GGT GACGTGTCTTCAAGAAGA-3'; si-NETO2\#1, 5'-GAUGGA AGUUGAUAAGGAAAU-3'; si-NETO2\#2, 5'-GAAUAG AUGAGACGGGUACAUAGG-3'.

Cell proliferation assay. The MTT assay was performed to assess cell proliferation. Transfected A549 or H1299 cells were seeded into 96 -well plates at a density of $2 \times 10^{3}$ cells/well. Cells were cultured for 24,48 and $72 \mathrm{~h}$ at $37^{\circ} \mathrm{C}$. MTT solution ( $5 \mathrm{mg} / \mathrm{ml}$; Sigma-Aldrich; Merck KGaA) were added into each well. Following incubation for $4 \mathrm{~h}$ at $37^{\circ} \mathrm{C}$, absorbance was measured at a wavelength of $490 \mathrm{~nm}$, using a microplate reader (BioTek Epoch; Agilent).

RNA immunoprecipitation (RIP). RIP was performed using the EZ-Magna RIP kit (MilliporeSigma), according to the manufacturer's instructions. A549 and H1299 cells were collected 
Table I. Association between SNHG17 expression and the clinicopathological characteristics of patients with lung adenocarcinoma $(n=50)$.

\begin{tabular}{|c|c|c|c|c|}
\hline \multirow[b]{2}{*}{ Characteristic } & \multirow[b]{2}{*}{$\begin{array}{l}\text { Number } \\
\text { of patients }\end{array}$} & \multicolumn{2}{|c|}{$\begin{array}{c}\text { SNHG17 } \\
\text { expression }\end{array}$} & \multirow[b]{2}{*}{ P-value } \\
\hline & & $\begin{array}{l}\text { Low } \\
(n=21)\end{array}$ & $\begin{array}{l}\text { High } \\
(n=29)\end{array}$ & \\
\hline Sex & & & & 0.793 \\
\hline Female & 18 & 8 & 10 & \\
\hline Male & 32 & 13 & 19 & \\
\hline Age, years & & & & 0.536 \\
\hline$<60$ & 26 & 12 & 14 & \\
\hline$\geq 60$ & 24 & 9 & 15 & \\
\hline TNM stage & & & & $0.027^{\mathrm{a}}$ \\
\hline $\mathrm{I} / \mathrm{II}$ & 23 & 14 & 9 & \\
\hline III/IV & 27 & 7 & 20 & \\
\hline EGFR mutation & & & & 0.089 \\
\hline No & 28 & 12 & 16 & \\
\hline Yes & 22 & 9 & 13 & \\
\hline PD-L1 expression & & & & $0.006^{\mathrm{b}}$ \\
\hline Low & 22 & 14 & 8 & \\
\hline High & 28 & 7 & 21 & \\
\hline
\end{tabular}

Data were analyzed using the $\chi^{2}$ test. ${ }^{\mathrm{a} P}<0.05$; ${ }^{\mathrm{b}} \mathrm{P}<0.01$. TNM, tumornode-metastasis; EGFR, epidermal growth factor receptor; PD-L1, programmed death ligand 1.

and lysed using RIP lysis buffer (MilliporeSigma). Cell lysis solutions were incubated with anti-Ago2 (cat. no. 2897; 1:50) or anti-IgG (cat. no. 6990S; 1:50) (both from Cell Signaling Technology, Inc.) conjugated with magnetic beads for $4 \mathrm{~h}$ at $4^{\circ} \mathrm{C}$. The immunoprecipitated RNA was harvested and detected via RT-qPCR analysis.

RNA pull-down assay. The biotin-labeled SNHG17 and no-biotin-labeled NC were synthesized by Shanghai GenePharma Co., Ltd., and cultured with $50 \mu \mathrm{l}$ M-280 Streptavidin magnetic beads (Invitrogen; Thermo Fisher Scientific, Inc.). The pcDNA3.1 vector (Invitrogen; Thermo Fisher Scientific, Inc.) was used to construct SNHG17 overexpression plasmids and transfected as aforementioned. The A549 or H1299 cells were lyzed with IP Lysis Buffer (Thermo Fisher Scientific, Inc.). Lysates $(50 \mu \mathrm{g})$ were incubated with probe-coated beads (Thermo Fisher Scientific, Inc.). The RNA-bound complex was subsequently eluted with Elution Buffer (Thermo Fisher Scientific, Inc.) and the isolation of completes was performed using a magnet. The contents of target RNA were detected using RT-qPCR.

Western blotting. The cells were lysed using RIPA buffer (Beyotime Institute of Biotechnology) and the protein concentration was measured using a BCA kit (Beyotime Institute of Biotechnology). Proteins (35 $\mu \mathrm{g}$ per lane) were separated using 10\% SDS-PAGE and transferred onto PVDF membranes
(MilliporeSigma). The membranes were blocked with 5\% skimmed milk at room temperature for $2 \mathrm{~h}$, then incubated with the following primary antibodies overnight at $4^{\circ} \mathrm{C}$ : E-cadherin [cat. no. 3195S; 1:1,000; Cell Signaling Technology, Inc. (CST)], $\beta$-catenin (cat. no. 8480S; 1:1,000; CST), vimentin (cat. no. 5741S; 1:1,000; CST), snail (cat. no. 3879S; 1:1,000; CST), twist (cat. no. 69366S; 1:1,000; CST), NETO2 (cat. no. ab109288; 1:1,000; Abcam) and $\beta$-actin (cat. no. 3700S; 1:10,000; CST). Following which, the membranes were incubated with the following HRP-conjugated secondary antibodies at room temperature for $1 \mathrm{~h}$ : Goat anti-rabbit (cat. no. ab205718; $1: 2,000$ ) and goat anti-mouse (cat. no. ab205719; 1:2,000) (both from Abcam). Protein bands were detected using ECL substrate (Thermo Fisher Scientific, Inc.).

Transwell assay. Transfected cells ( $5 \times 10^{4}$ cells) were seeded into the upper chambers of Transwell plates (Corning, Inc.) in culture medium without FBS. RPMI-1640 medium (Gibco; Thermo Fisher Scientific, Inc.) supplemented with 10\% FBS was plated in the lower chambers. The upper chambers of Transwell plates were coated with and without Matrigel $\left(37^{\circ} \mathrm{C}\right.$ for $8 \mathrm{~h}$ ) for the invasion and migration assays, respectively. After incubation for $24 \mathrm{~h}$ at $37^{\circ} \mathrm{C}$, the invasive cells were fixed with $4 \%$ paraformaldehyde for $10 \mathrm{~min}$ and subsequently stained with $0.1 \%$ crystal violet for $10 \mathrm{~min}$, both at room temperature. Images of the stained cells were captured from five randomly selected fields using an inverted fluorescence microscope (magnification $\mathrm{x} 400$ ).

Target prediction and bioinformatics. The target proteins of miR-193a-5p were predicted using the online databases, TargetScan (http://www.targetscan.org/vert_71/), miRDB (http://mirdb.org/) and StarBase3.0 (http://starbase.sysu. edu.cn). Correlation between SNHG17 and miR-193a-5p expression levels in patients with LUAD was performed using StarBase3.0. Specifically, the Pan-cancer option was selected, then the RNA-RNA co-expression module for LUAD. miR-193a-5p expression level in LUAD was evaluated using Tumor-miRNA-Pathway (http://bioinfo.life.hust.edu. cn/miR_path/index.html) and StarBase3.0 databases. For the tumor-miRNA-pathway database, miR-193a-5p expression level was obtained by selecting miR-193a-5p and LUAD. For the StarBase3.0 database, the Pan-cancer option was selected, then the miRNA differential expression module for miR-193a-5p in LUAD. NETO2 expression level was evaluated using StarBase3.0 and UALCAN databases (http://ualcan. path.uab.edu). NETO2 expression in LUAD was analyzed by selecting Pan-cancer, then the gene differential expression module in the StarBase3.0 database. In the UALCAN database, the expression level of NETO2 was obtained by entering NETO2 and selecting LUAD in TCGA module.

Dual-luciferase reporter assay. The wild-type (WT) or mutant (MUT) reporter plasmids of SNHG17 and NETO2 were constructed by cloning WT or MUT sequences into pGL3 luciferase reporter plasmids (Promega Corporation). The $293 \mathrm{~T}$ cells $\left(1 \times 10^{5} /\right.$ well $)$ were seeded into a 24 -well plate and co-transfected with SNHG17-WT, SNHG17-MUT, NETO2-WT or NETO2-MUT reporter plasmids, and the aforementioned miR-193a-5p mimics or mimics NC, using Lipofectamine ${ }^{\circledR} 3000$ 
A

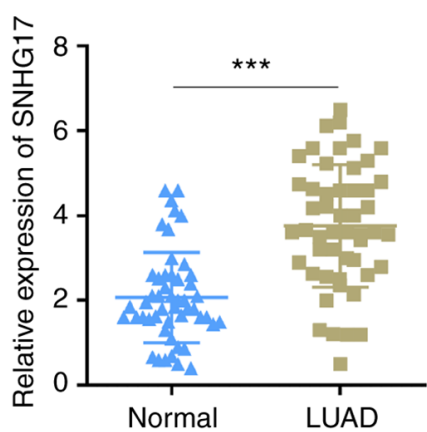

$\mathrm{D}$

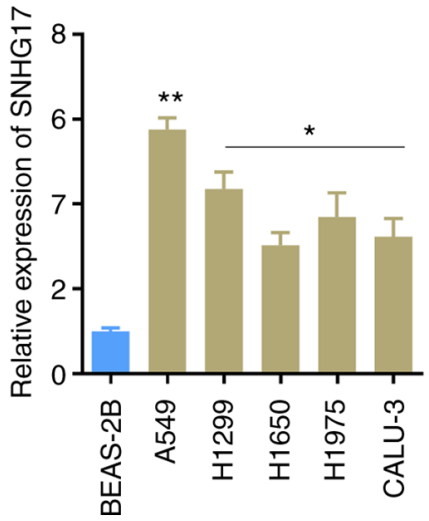

$\mathrm{B}$
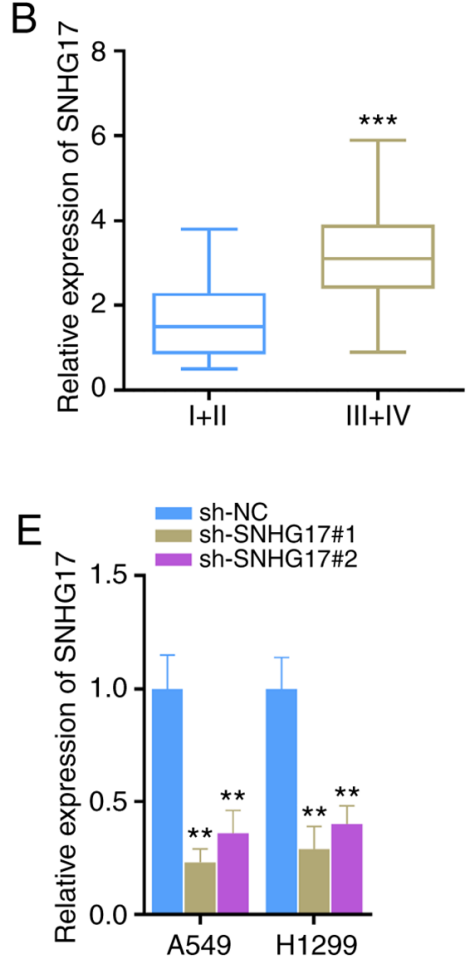

C
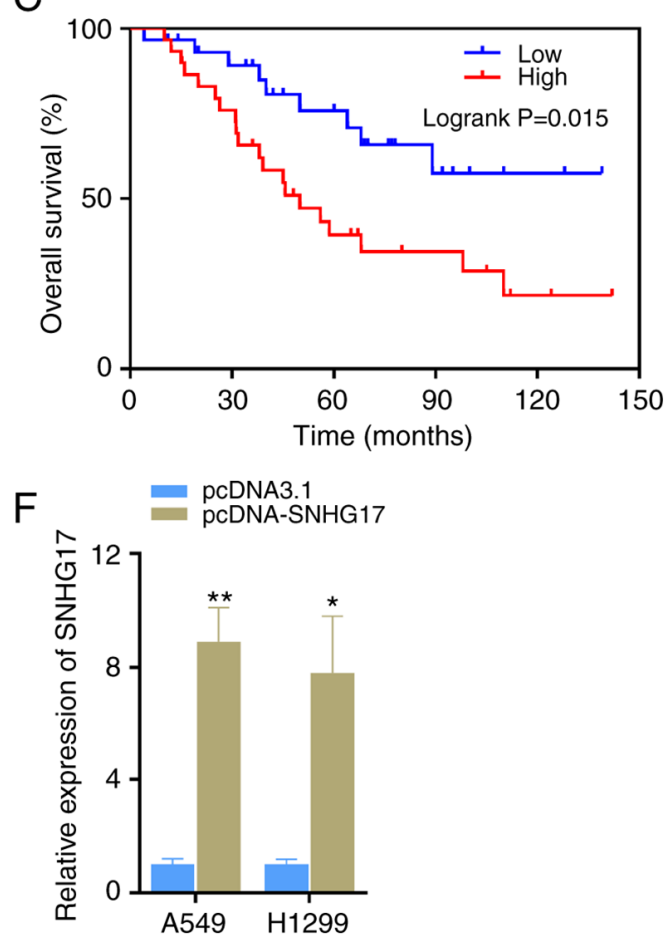

Figure 1. SNHG17 expression is upregulated in LUAD. (A) SNHG17 expression in normal lung tissues ( $\mathrm{n}=50$ ) and lung cancer tissues ( $\mathrm{n}=50$ ). ${ }^{* * *} \mathrm{P}<0.001$. (B) SNHG17 expression at different stages of LUAD; I/II ( $\mathrm{n}=23)$, III/IV ( $\mathrm{n}=27)$. ${ }^{* * *} \mathrm{P}<0.001$. (C) Kaplan-Meier survival analysis was performed to detect the survival time of patients with LUAD, based on SNHG17 expression. (D) Reverse transcription-quantitative PCR analysis was performed to detect SNHG17 expression in normal BEAS-2B lung cells and five LUAD cell lines. ${ }^{*} \mathrm{P}<0.05,{ }^{* *} \mathrm{P}<0.01$ vs. BEAS-ZB. (E) A549 and H1299 cells were transfected with sh-NC, sh-SNHG17\#1 or sh-SNHG17\#2, and SNHG17 expression was detected. ${ }^{* *} \mathrm{P}<0.01$ vs. sh-NC. (F) SNHG17 expression increased in A549 and H1299 cells following transfection with pcDNA-SNHG17. ${ }^{*} \mathrm{P}<0.05,{ }^{* *} \mathrm{P}<0.01$. LUAD, lung adenocarcinoma; sh, short hairpin; NC, negative control.

reagent (Invitrogen; Thermo Fisher Scientific, Inc.). Luciferase activities were detected using the Dual-Luciferase Reporter Assay System (Promega Corporation) and normalized to Renilla luciferase activities at $24 \mathrm{~h}$ post-transfection.

Statistical analysis. Statistical analysis was performed using GraphPad Prism 8 (GraphPad Software, Inc.). Data are presented as the mean \pm SD. All the experiments were repeated three times. Student's t-test was used to compare differences between two groups, while one-way ANOVA followed by Tukey's post hoc test were used to compare differences between multiple groups. Kaplan-Meier survival analysis was used to analyze the effect of SNHG17 expression on survival time in patients with LUAD and the log-rank test was used to determine statistical significance. According to the median of SNHG17 expression level, the patients were divided into the high and low expression level groups. Association between SNHG17 expression and the clinicopathological characteristics of patients with lung adenocarcinoma was analyzed using a $\chi^{2}$ test. The correlation of gene expression was analyzed using linear regression analysis. $\mathrm{P}<0.05$ was considered to indicate a statistically significant difference.

\section{Results}

SNHG17 expression is upregulated in LUAD tissues and cells. To determine the role of SNHG17 in LUAD progression,
RT-qPCR analysis was performed to detect SNHG17 expression in LUAD cancer tissues and adjacent normal tissues. The results demonstrated that SNHG17 expression was significantly upregulated in cancer tissues compared with that in normal tissues (Fig. 1A). In addition, SNHG17 expression gradually increased with cancer progression. Furthermore, SNHG17 expression was significantly higher in stages III and IV of LUAD compared with stages I and II (Fig. 1B). Taken together, these results suggest that SNHG17 expression was associated with LUAD progression. Kaplan-Meier survival analysis demonstrated that patients with LUAD and high SNHG17 expression level had poor prognosis (Fig. 1C). Consistently, SNHG17 expression was significantly higher in the LUAD cell lines compared with that in the normal cell line (Fig. 1D). Among these cell lines, SNHG17 expression was the highest in A549 and H1299 cells, thus these cell lines were selected for subsequent experimentation. A total of two shRNAs (sh-SNHG17\#1 and sh-SNHG17\#2) were synthesized to knockdown SNHG17 expression in the A549 and H1299 cells and the results showed that SNHG17 expression level was reduced (Fig. 1E). On the contrary, SNHG17 expression was significantly increased in cells transfected with overexpression plasmid of SNHG17 (Fig. 1F). Collectively, these results suggest that SNHG17 expression is upregulated in LUAD cancer tissues and cell lines.

SNHG17 facilitates the malignant phenotypes of LUAD cells. In the migration and invasion assays, transfection with shRNAs significantly inhibited the migratory and invasive 
A
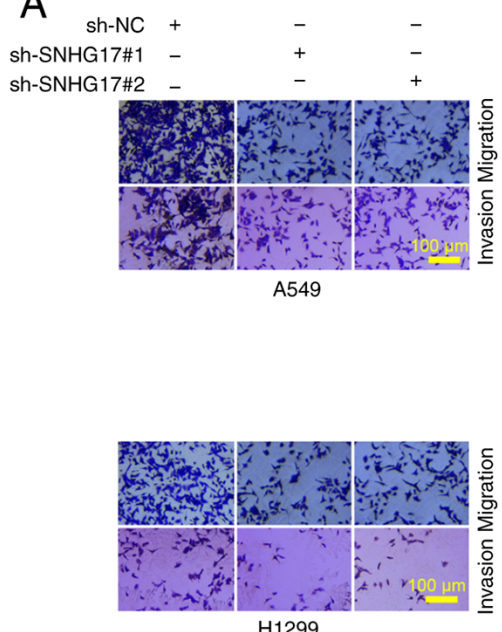

H1299
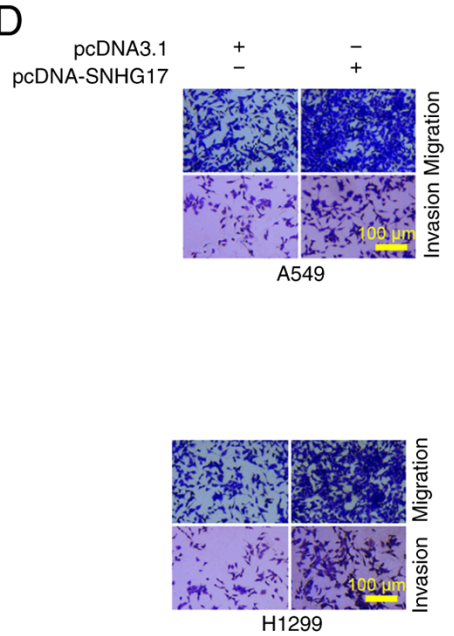
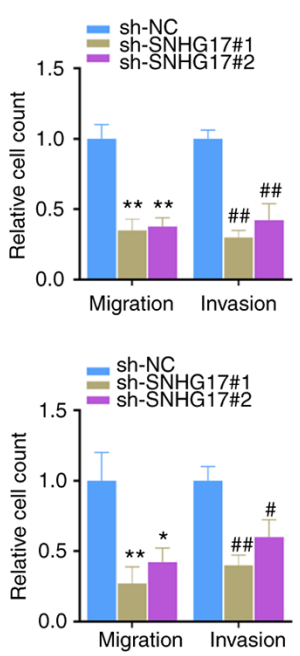

B
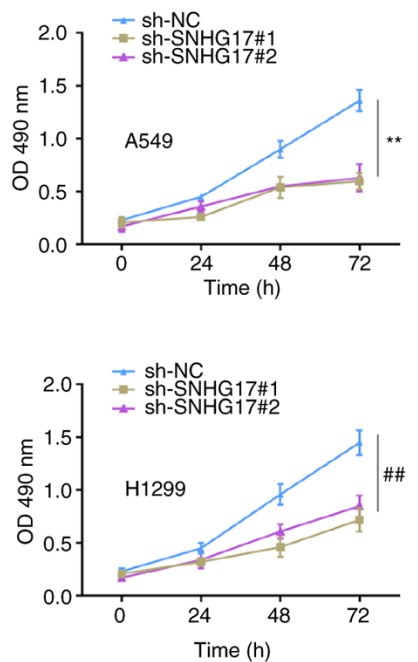

E
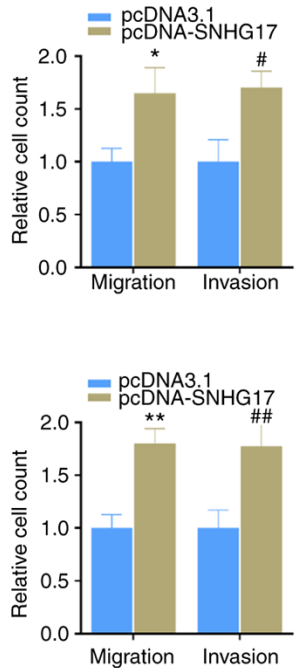

C
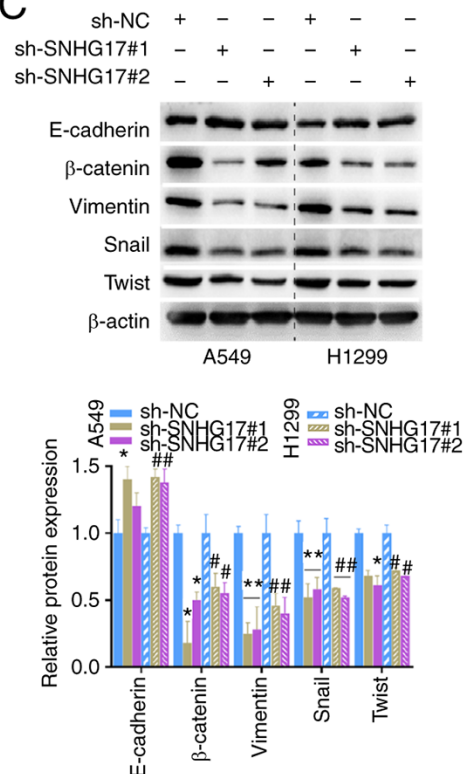

F
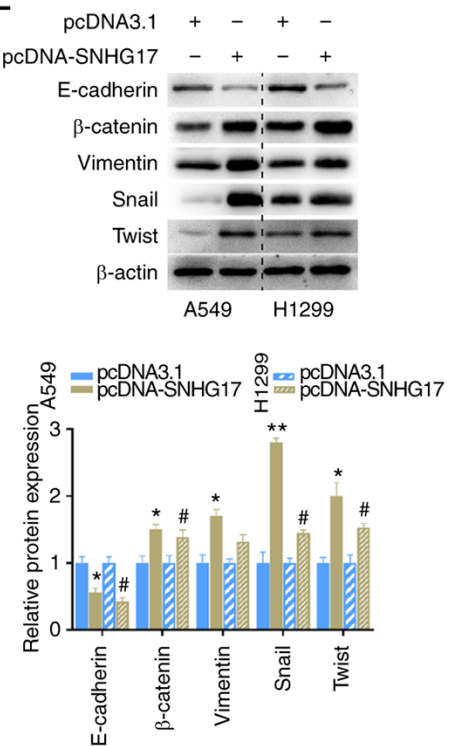

Figure 2. Regulation of SNHG17 on the migration, invasion, proliferation and EMT of A549 and H1299 cells. (A) Migration and invasion analyses of A549 and H1299 cells transfected with sh-NC, sh-SNHG17\#1 or sh-SNHG17\#2. ${ }^{*} \mathrm{P}<0.05,{ }^{* *} \mathrm{P}<0.01$ vs. sh-NC; ${ }^{*} \mathrm{P}<0.05,{ }^{\# \prime} \mathrm{P}<0.01$ vs. sh-NC. (B) The MTT assay was performed to assess cell proliferation in the indicated groups. ${ }^{* *} \mathrm{P}<0.01$ vs sh-NC; ${ }^{\# \#} \mathrm{P}<0.01$ vs. sh-NC. (C) Western blot analysis was performed to detect the protein expression levels of the EMT markers following SNHG17 knockdown. " $\mathrm{P}<0.05,{ }^{* *} \mathrm{P}<0.01$ vs. A549 sh-NC; ${ }^{~} \mathrm{P}<0.05$, ${ }^{\# \#} \mathrm{P}<0.01$ vs. H1299 sh-NC. (D) Migration and invasion analyses of A549 and H1299 cells following overexpression of SNHG17. ${ }^{*} \mathrm{P}<0.05,{ }^{* *} \mathrm{P}<0.01$ vs. pcDNA3.1; $\mathrm{P}<0.05$, ${ }^{\# \#} \mathrm{P}<0.01$ vs. pcDNA3.1. (E) The MTT assay was performed to assess the proliferation of A549 and H1299 cells following transfection with pcDNA3.1 and pcDNA-SNHG17. ${ }^{* *} \mathrm{P}<0.01$ vs. pcDNA3.1; ${ }^{* \#} \mathrm{P}<0.01$ vs. pcDNA3.1. (F) Western blot analysis was performed to detect the protein expression levels of the EMT markers following overexpression of SNHG17. $\mathrm{P}<0.05,{ }^{* *} \mathrm{P}<0.01$ vs. A549 pcDNA3.1; ${ }^{*} \mathrm{P}<0.05$ vs. H1299 pcDNA3.1. EMT, epithelial-to-mesenchymal transition; sh, short hairpin; NC, negative control.

abilities of A549 cells (Fig. 2A). Similar results were also observed in H1299 cells (Fig. 2A). The results of the MTT cell proliferation assay demonstrated that cells transfected with sh-SNHG17 exhibited a significantly slower proliferative ability (Fig. 2B). E-cadherin, $\beta$-catenin, vimentin, snail and twist are EMT-related proteins. In the process of EMT, the expression levels of $\beta$-catenin, vimentin, snail and twist increase, while E-cadherin expression decrease (23-25). Following SNHG17 knockdown in the present study, the protein expression levels of $\beta$-catenin, vimentin, snail and twist decreased, while E-cadherin expression increased in A549 and H1299 cells (Fig. 2C). Notably, these effects were reversed following overexpression of SNHG17. The overexpression plasmid of SNHG17 was constructed and transfected into A549 and H1299 cells. Transwell and MTT assays were performed to detect cell proliferation, migration and invasion. The results demonstrated that overexpression of SNHG17 significantly promoted the migration, invasion and proliferation of A549 and H1299 cells (Fig. 2D and E). In addition, overexpression of SNHG17 also increased the protein expression level of EMT-related proteins in the LUAD cells (Fig. 2F). Taken together, these results suggest that overexpression of SNHG17 accelerates cancer cell migration, invasion and proliferation, and the EMT process. 
A
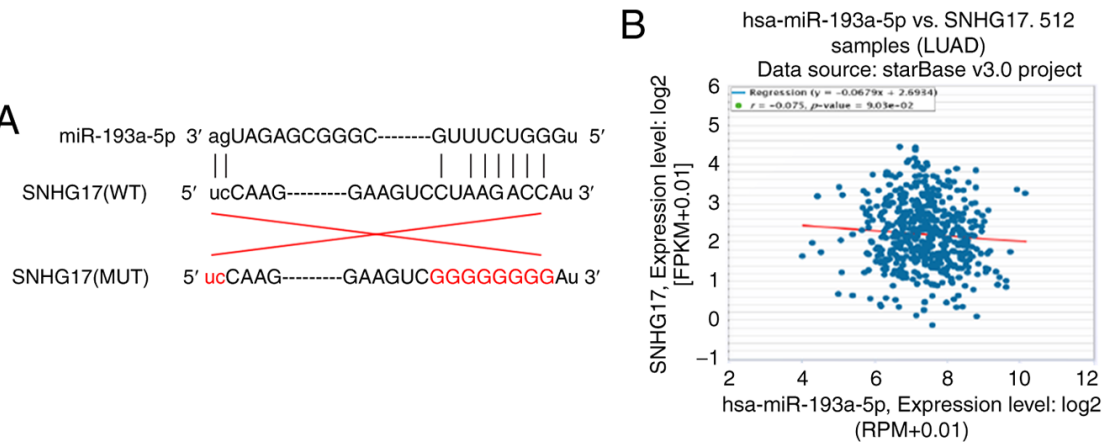

D

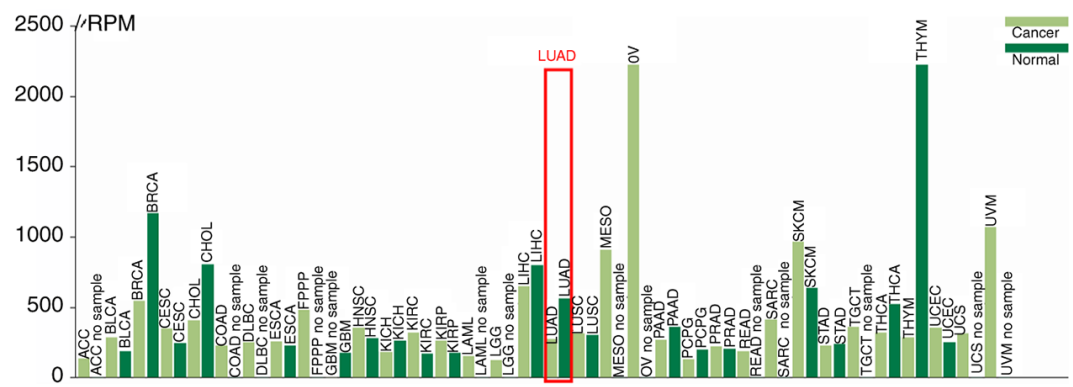

C

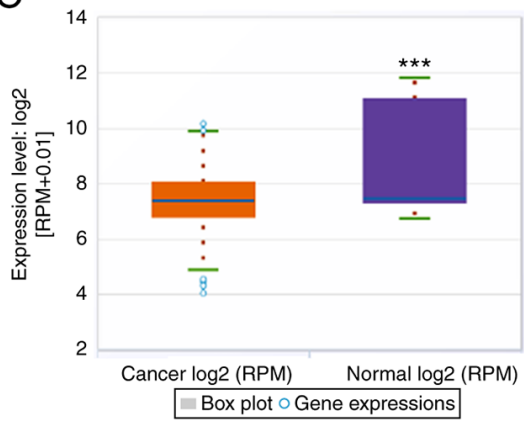

F

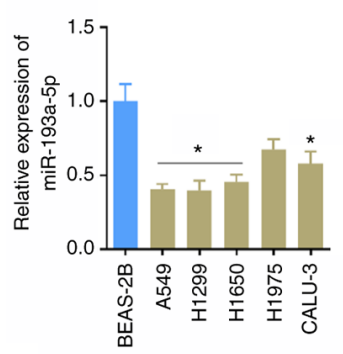

I

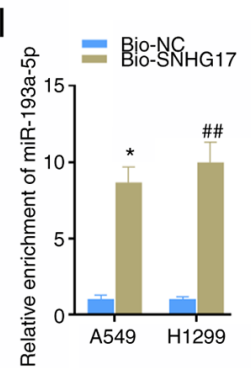

G

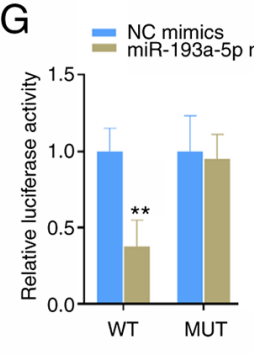

$\mathrm{H}$

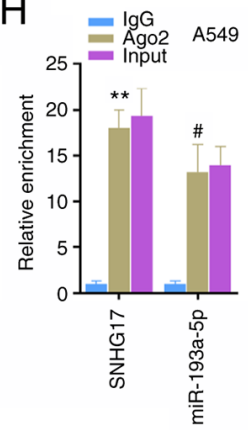

E

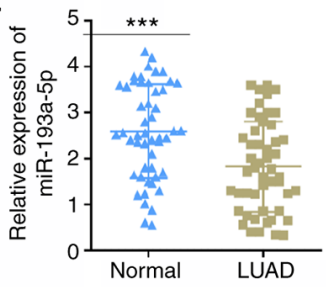

$\mathrm{J}$

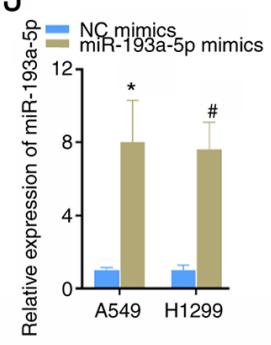

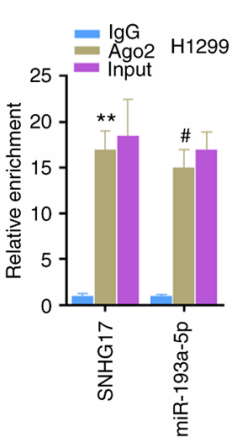

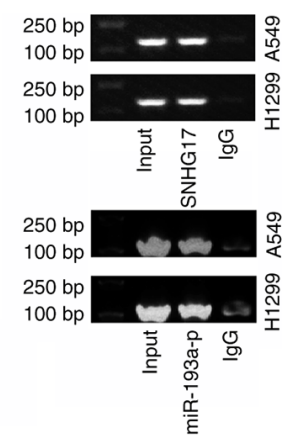

$\mathrm{K}$
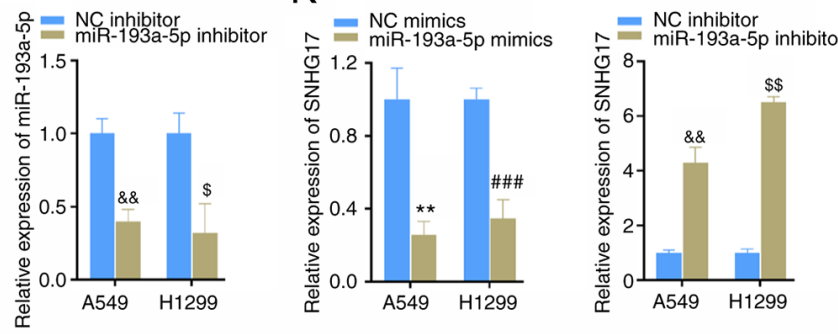

L

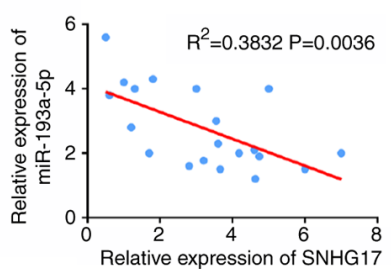

Figure 3. miR-193a-5p is a target of SNHG17. (A) Bioinformatics analysis indicated the potential binding sites between SNHG17 and miR-193a-5p. (B) Correlation analysis indicated a negative correlation between miR-193a-5p and SNHG17 expression in LUAD tissues, according to the StarBase database. (C) miR-193a-5p expression was downregulated in LUAD, according to the StarBase database. ${ }^{* * *} \mathrm{P}<0.001$. (D) miR-193a-5p expression analysis in cancers was obtained from the Tumor-miRNA-Pathway database. (E) Reverse transcription-quantitative PCR analysis was performed to detect miR-193a-5p expression in LUAD tissues and adjacent normal tissues. ${ }^{* * *} \mathrm{P}<0.001$. (F) miR-193a-5p expression was detected in normal and LUAD cell lines. ${ }^{*} \mathrm{P}<0.05$ vs. BEAS-ZB (G) The dual-luciferase reporter assay was performed to verify the association between SNHG17 and miR-193a-5p expression. ${ }^{* *} \mathrm{P}<0.01$ vs. NC mimics. (H) The interaction between SNHG17 and miR-193a-5p was assessed using the RIP assay. ${ }^{* *} \mathrm{P}<0.01 \mathrm{vs}$. IgG; ${ }^{*} \mathrm{P}<0.05$, IgG. (I) The enrichment of miR-193a-5p was assessed in A549 and H1299 cells via the RNA pull down assay. ${ }^{*} \mathrm{P}<0.05$ vs. Bio-NC; ${ }^{\# \#} \mathrm{P}<0.05$ vs. Bio-NC. (J) miR-193a-5p expression level in cells transfected mimics or inhibitor. ${ }^{*} \mathrm{P}<0.05$ vs. NC mimics, ${ }^{\&}{ }^{\&} \mathrm{P}<0.01$ vs. NC inhibitor; ${ }^{~} \mathrm{P}<0.05$ vs. NC mimics, ${ }^{\$} \mathrm{P}<0.05$ vs. NC inhibitor. (K) SNHG17 expression level in

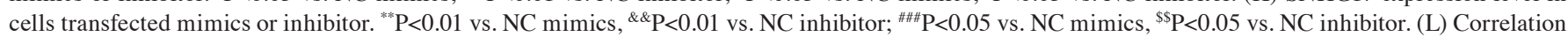
analysis was performed to detect the correlation between miR-193a-5p and SNHG17 expression. miR, microRNA; LUAD, lung adenocarcinoma; NC, negative control; WT, wild-type; MUT, mutant. 

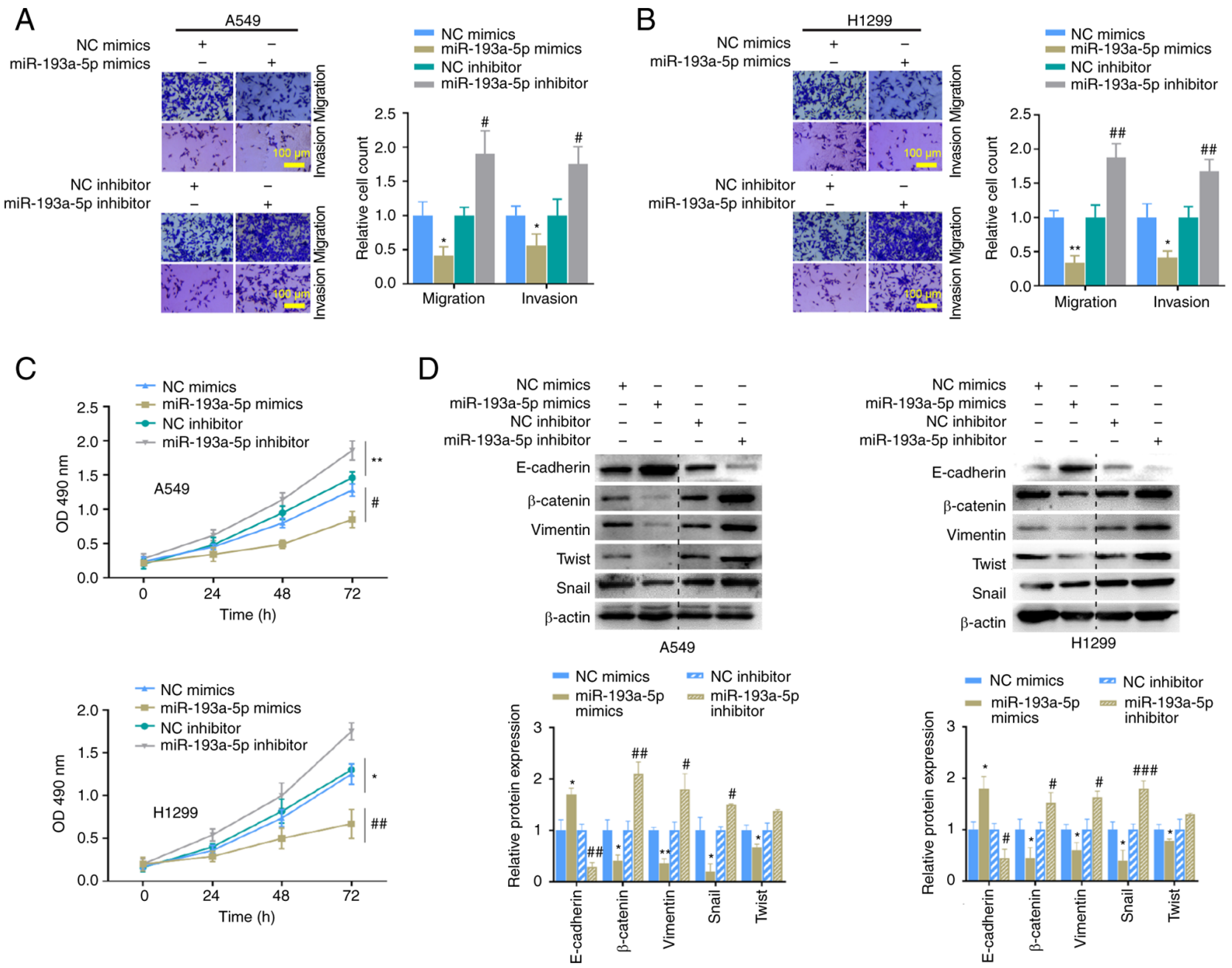

Figure 4. miR-193a-5p regulates migration, invasion, proliferation and EMT of A549 and H1299 cells. Migration and invasion analyses of (A) A549 and (B) H1299 cells transfected with miR-193a-5p mimics or inhibitor. (C) The MTT assay was performed to assess the proliferation of A549 or H1299 cells transfected with miR-193a-5p mimics or inhibitor.(D) The expression levels of the EMT markers were detected in A549 or H1299 cells transfected with miR-193a-5p mimics or inhibitor. ${ }^{*} \mathrm{P}<0.05,{ }^{* *} \mathrm{P}<0.01$ vs. NC mimics; ${ }^{~} \mathrm{P}<0.05,{ }^{\# \#} \mathrm{P}<0.01,{ }^{\# \# "} \mathrm{P}<0.001$ vs. NC inhibitor. miR, microRNA; EMT, epithelial-to-mesenchymal transition; $\mathrm{NC}$, negative control; $\mathrm{OD}$, optical density.

SNHG17 can directlytarget miR-193a-5pandinhibit its expression. It is well-known that lncRNAs can sponge miRNAs and inhibit their expression and functions (26). To further investigate the function of SNHG17 in LUAD, the present study predicted its downstream genes and miR-193a-5p was selected for subsequent experimentation due to the lower expression of miR-193a-5p in some types of cancer, for example, hepatocellular carcinoma, colon cancer and non-small cell lung cancer (27-29). The potential binding sequence between SNHG17 and miR-193a-5p, and mutated binding sequence of SNHG17 are presented in Fig. 3A. Correlation analysis revealed the negative correlation between SNHG17 and miR-193a-5p expression in patients with LUAD, using the StarBase database (Fig. 3B). The results also demonstrated that miR-193a-5p expression was significantly downregulated in LUAD cancer tissues compared with that in normal tissues, using the StarBase database (Fig. 3C). These results were confirmed following analysis of The Cancer Genome Atlas expression profile, using Tumor-miRNA-Pathway (http://bioinfo.life.hust edu.cn/miR_path/index.html) (Fig. 3D). miR-193a-5p expression was subsequently detected in LUAD cancer tissues and cells. Consistent with the bioinformatics analysis, the results demonstrated that miR-193a-5p expression was significantly downregulated in cancer tissues and cells (Fig. 3E and F). The dual-luciferase reporter assay was performed to confirm whether SNHG17 can directly target and regulate miR-193a-5p expression.

The luciferase reporter vectors, including WT or MUT binding sequences were constructed and co-transfected with miR-193a-5p mimics into 293T cells. The luciferase activity of WT reporter vectors was significantly inhibited following transfection with miR-193a-5p mimics, while no differences were observed between the MUT groups (Fig. 3G). The RIP and RNA pull down assays were performed to assess the interaction between SNHG17 and miR-193a-5p and the results showed the significant interaction and enrichment between SNHG17 and miR-193a-5p (Fig. 3H and I). Furthermore, the present study detected the knockdown or overexpression effects of miR-193a-5p on SNHG17 in LUAD cells (Fig. 3J) and SNHG17 expression was negatively regulated by miR-193a-5p in cancer cells (Fig. 3K). Furthermore, correlation analysis confirmed the negative correlation between SNHG17 and 
A

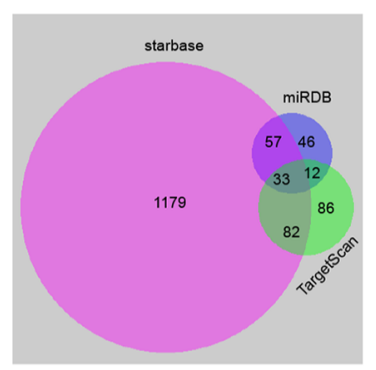

C
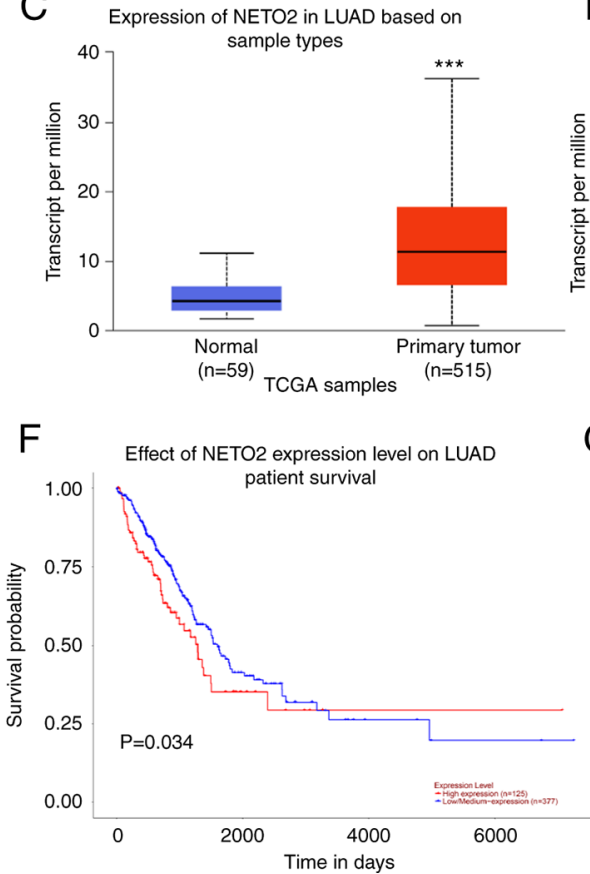

B

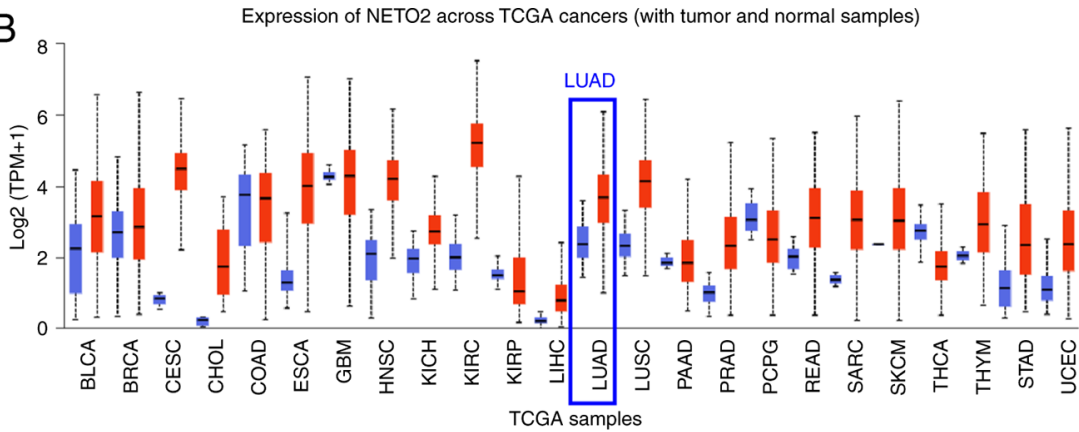

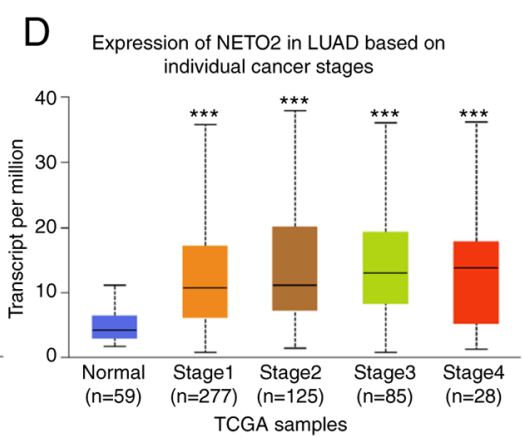

G

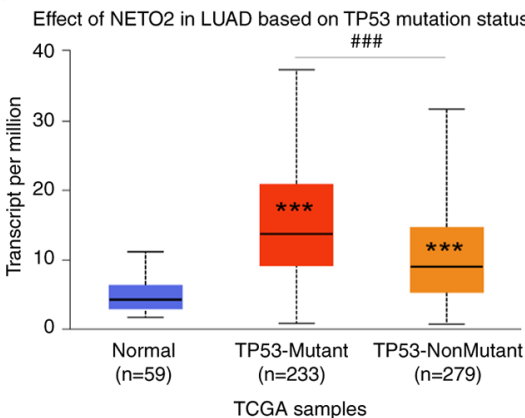

E Expression of NETO2 in LUAD based

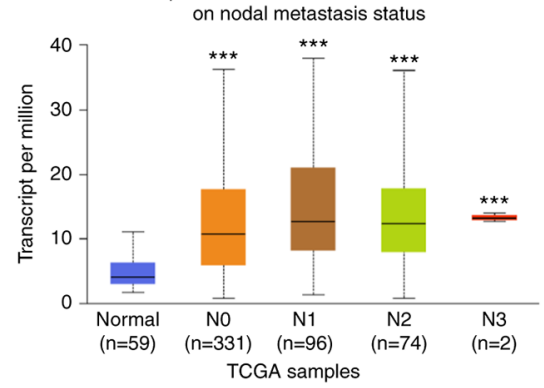

$\mathrm{H}$

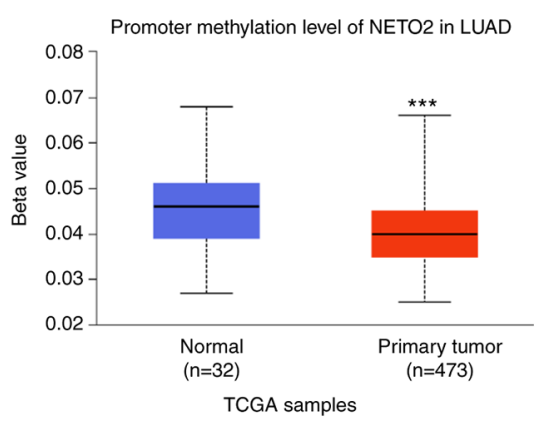

Figure 5. NETO2 acts as an oncogene in LUAD. (A) The 33 potential downstream target proteins of miR-193a-5p were predicted using the StarBase, TargetScan and miRDB databases. NETO2, one of the 33 target genes, was further studied in LUAD. The expression and potential function of NETO2 were analyzed, according to UALCAN. (B) Expression of NETO2 across TCGA cancers. (C) Expression of NETO2 in LUAD cancer tissues and normal tissues. ${ }^{* * *}$ P $<0.001$. (D) Expression of NETO2 in different cancer stages of LUAD. ${ }^{* * * *} \mathrm{P}<0.001$ vs. normal. (E) Association between NETO2 and nodal metastasis status of patients with LUAD. ${ }^{* * * *} \mathrm{P}<0.001$ vs. normal. (F) Survival analysis of patients with LUAD based on NETO2 expression. (G) NETO2 expression increased in the presence of TP53 mutation. ${ }^{* * *} \mathrm{P}<0.001$ vs. normal; ${ }^{\# \# t} \mathrm{P}<0.001$ vs. TP53-Mutant. $(\mathrm{H})$ The reduced promoter methylation level of NETO2 in LUAD. ${ }^{* * *} \mathrm{P}<0.001$. NETO2, neuropilin and tolloid-like 2; LUAD, lung adenocarcinoma; miR, microRNA; TCGA, The Cancer Genome Atlas.

miR-193a-5p in LUAD tissues (Fig. 3L). Taken together, these results suggest that miR-193a-5p is a direct target of SNHG17, and its expression is negatively correlated with SNHG17 expression in LUAD.

miR-193a-5p regulates the migration, invasion, proliferation and EMT of LUAD cells. To investigate the effects of miR-193a-5p on LUAD progression in vitro, A549 and H1299 cells were transfected with miR-193a-5p mimics, inhibitor, or their respective NCs to implement Transwell, MTT and western blot assays. The results demonstrated that overexpression of miR-193a-5p significantly suppressed cell migration, invasion (Fig. 4A and B) and proliferation (Fig. 4C). Notably, E-cadherin expression increased, while the expression levels of $\beta$-catenin, vimentin, twist and snail decreased following transfection with miR-193a-5p mimics (Fig. 4D). Conversely, cell migration, invasion and proliferation, and EMT were significantly promoted in A549 and H1299 cells following transfection with miR-193a-5p inhibitor (Fig. 4A-D). Collectively, these results suggest that miR-193a-5p is negatively involved in the malignant progression of LUAD in vitro.

NETO2 is an oncogene in LUAD and is inhibited bymiR-193a-5p . The downstream target proteins of miR-193a-5p were predicted via bioinformatics analysis. Among the 33 potential target genes (Fig. 5A) and combined with the discovery in UALCAN database, NETO2 was revealed to be highly expressed in different types of human cancer, such as cervical squamous cell carcinoma and endocervical, LUAD and lung squamous cell carcinoma (Fig. 5B). The present study subsequently analyzed the expression and potential functions of NETO2 in LUAD. The results demonstrated that NETO2 expression was significantly upregulated in LUAD primary tumor tissues compared with normal tissues (Fig. 5C). In addition, the LUAD cancer stages were positively correlated with NETO2 expression (Fig. 5D). Notably, patients with lymph node metastasis had higher NETO2 expression (Fig. 5E). Furthermore, high NETO2 

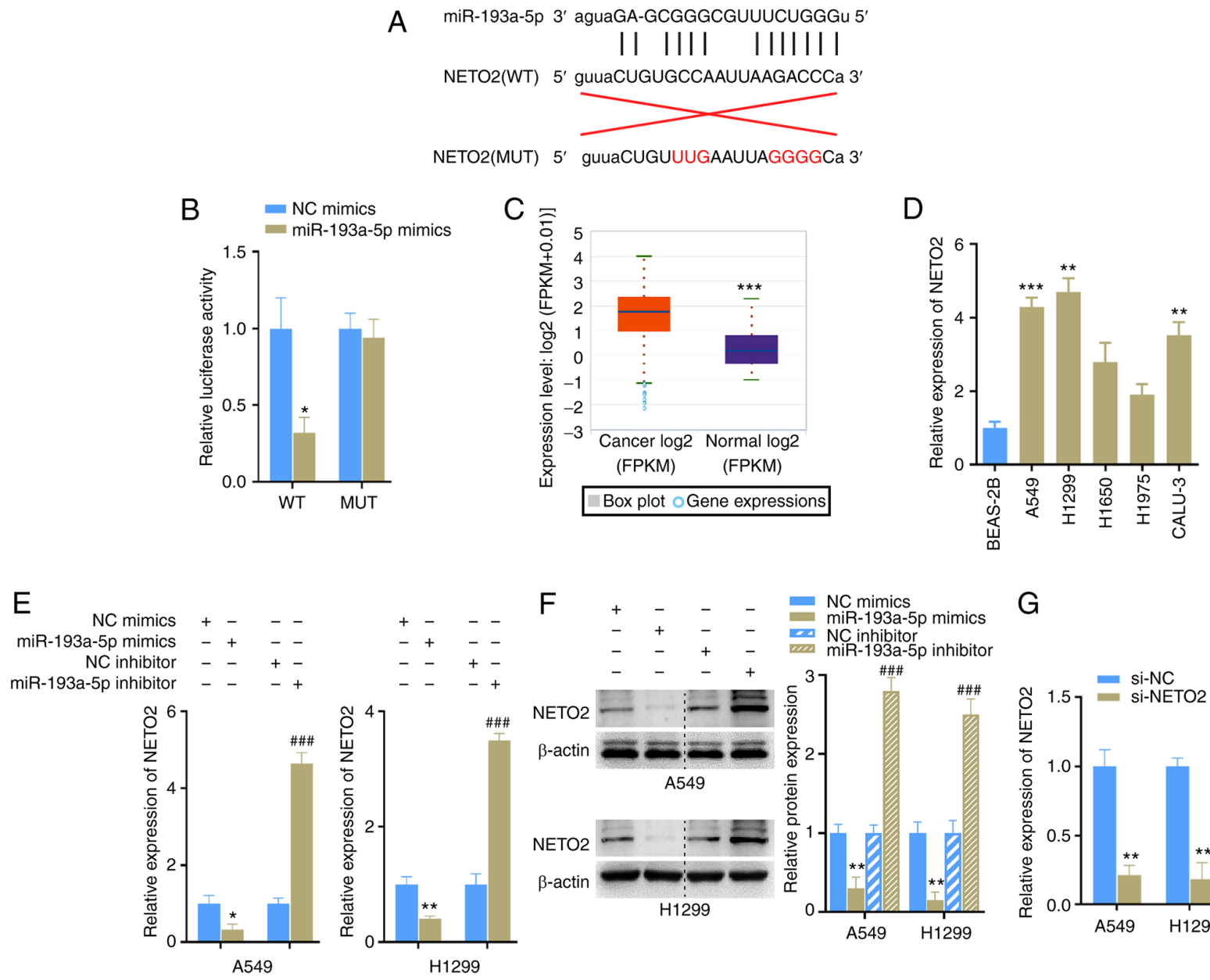

$\begin{array}{rlllll}\mathrm{F} & + & - & - & - & \mathrm{N} \text { mimics } \\ & - & + & - & - & \text { miR-193a-5p mimics } \\ \text { NC inhibitor } & - & - & + & - & \text { miR-193a-5p inhibitor }\end{array}$
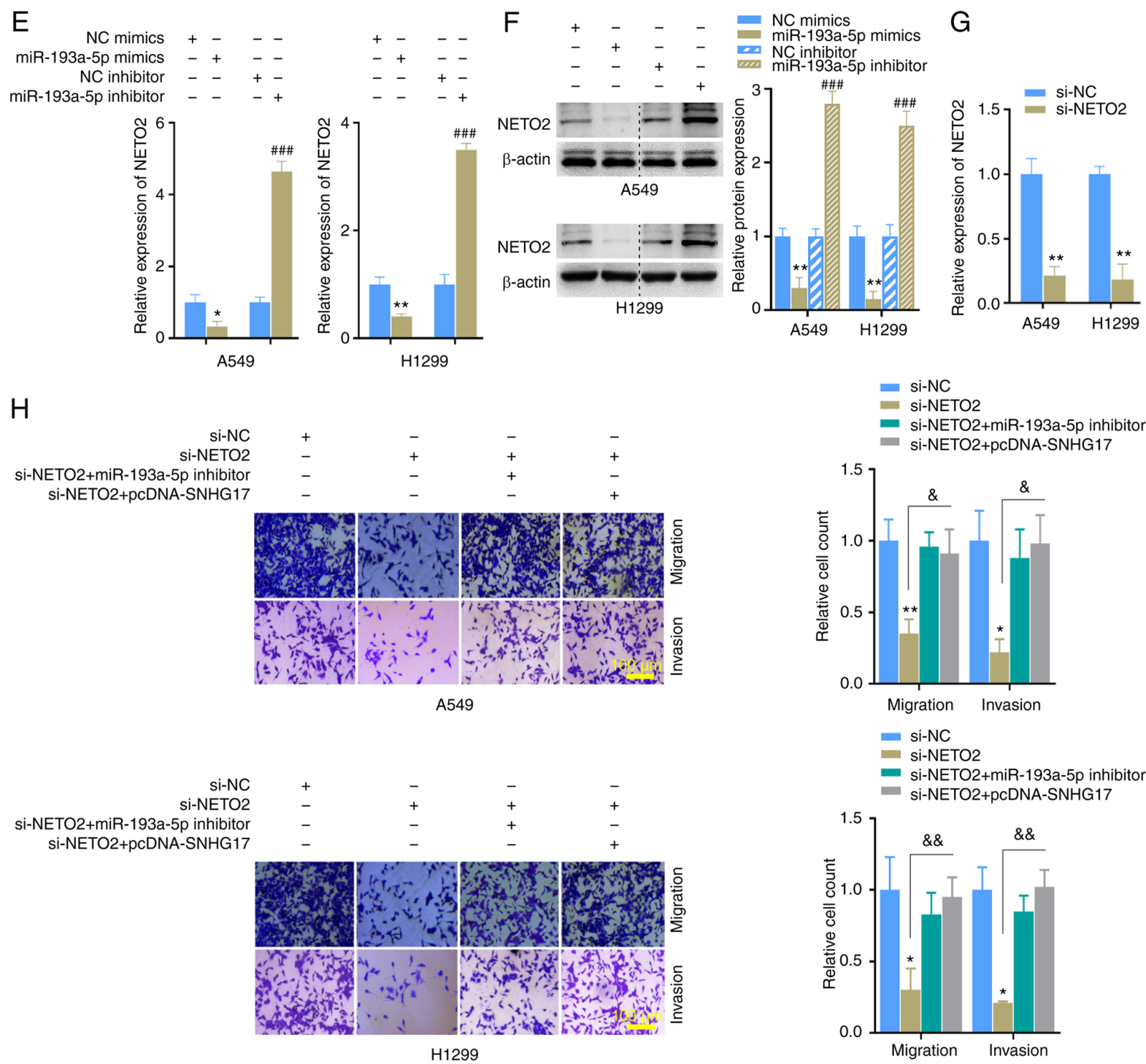

Figure 6. miR-193a-5p regulates NETO2 expression and the functions of NETO2 are affected by miR-193a-5p and SNHG17. (A) The potential binding and mutation sites between NETO2 and miR-193a-5p. (B) The dual-luciferase reporter assay revealed that NETO2 is directly targeted by miR-193a-5p. ${ }^{*} \mathrm{P}<0.05$ vs. NC mimics. (C) NETO2 expression was upregulated in LUAD cancer samples, according to the StarBase database. ${ }^{* * * *} \mathrm{P}<0.001$. (D) NETO2 expression was significantly upregulated in LUAD cells. ${ }^{* * *} \mathrm{P}<0.01,{ }^{* * *} \mathrm{P}<0.001$ vs. BEAS-2B. (E and F) Transfection with miR-193a-5p mimics or inhibitor regulated NETO2 mRNA and protein expression levels, respectively. $\mathrm{P}<0.05,{ }^{* *} \mathrm{P}<0.01$ vs. NC mimics; ${ }^{* \# \#} \mathrm{P}<0.001$ vs. NC inhibitor. (G) Knockdown result of NETO2. ${ }^{* *} \mathrm{P}<0.01$ vs. si-NC. (H) NETO2 knockdown inhibited the migratory and invasive abilities of A549 and H1299 cells, the effects of which were reversed following transfection with miR-193a-5p inhibitor or pcDNA-SNHG17. ${ }^{*} \mathrm{P}<0.05$, ${ }^{* * *} \mathrm{P}<0.01$ vs. si-NC; ${ }^{\&} \mathrm{P}<0.05$, ${ }^{\&}{ }^{\&} \mathrm{P}<0.01$ vs. si-NETO2. miR, microRNA; LUAD, lung adenocarcinoma; NC, negative control; si, small interfering; WT, wild-type; MUT, mutant. 

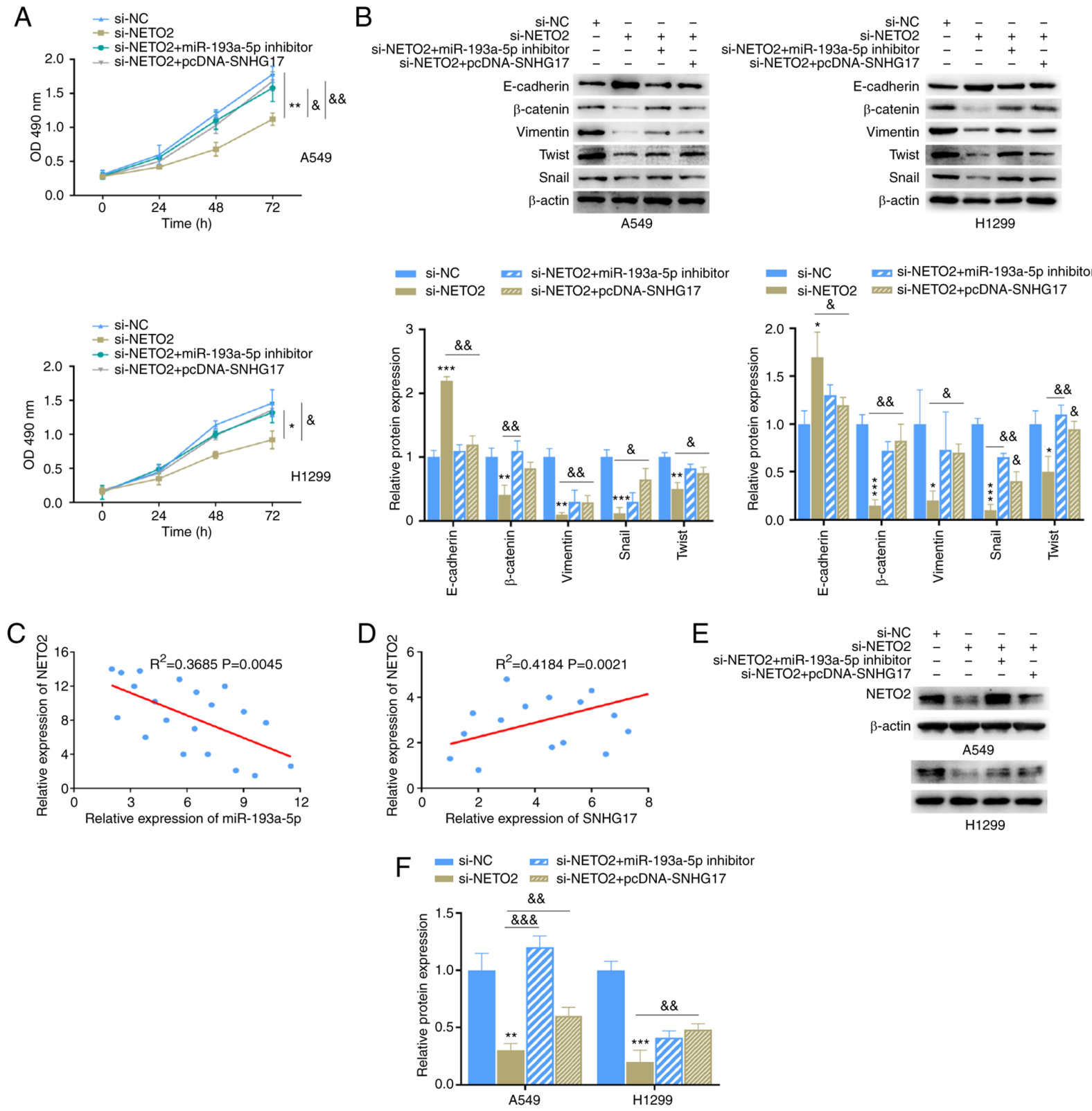

Figure 7. NETO2 function is affected by miR-193a-5p and SNHG17. (A) The proliferative ability of A549 and H1299 cells was assessed following transfection with the indicated groups. ${ }^{*} \mathrm{P}<0.05,{ }^{* *} \mathrm{P}<0.01$ vs. si-NC; ${ }^{\circledR} \mathrm{P}<0.05$, ${ }^{\&} \& \mathrm{P}<0.01$ vs. si-NETO2. (B) Western blot analysis was performed to detect the protein expression levels of the epithelial-to-mesenchymal markers following transfection with the indicated groups. ${ }^{*} \mathrm{P}<0.05,{ }^{* *} \mathrm{P}<0.01,{ }^{* * *} \mathrm{P}<0.001 \mathrm{vs}$. si-NC; ${ }^{\circledR} \mathrm{P}<0.05$, \&\&P $<0.01$ vs. si-NETO2. (C) Correlation analysis between NETO2 and miR-193a-5p expression. (D) Correlation analysis between NETO2 and SNHG17 expression. (E and F) The effects of miR-193a-5p and SNHG17 on NETO2 expression. ${ }^{* *} \mathrm{P}<0.01,{ }^{* * * *} \mathrm{P}<0.001$ vs. si-NC; \&\& $\mathrm{P}<0.01$, \&\&\& $\mathrm{P}<0.001$ vs. si-NETO2. miR, microRNA; si, small interfering; NC, negative control; OD, optical density.

expression predicted a poor prognosis in patients with LUAD (Fig. 5F). TP53 mutation is often involved in the occurrence and development of different types of cancer (30). In the present study, NETO2 expression was elevated in LUAD when the TP53 gene was mutated (Fig. 5G). Furthermore, promoter methylation of NETO2 significantly decreased in LUAD (Fig. 5H), which suggests that NETO2 expression is upregulated in LUAD and may further lead to the occurrence and development of LUAD.

miR $-193 a-5 p$ regulates NETO2 expression in LUAD cells. The role of NETO2 in LUAD cells was investigated. The binding sequence between miR-193a-5p and NETO2 was predicted (Fig. 6A) and the dual-luciferase assay was performed.
The results demonstrated that the luciferase activity of the NETO2-WT reporter was significantly inhibited following transfection with miR-193a-5p mimics, whereas no significant difference was observed between the NETO2-MUT groups (Fig. 6B). High NETO2 mRNA expression was identified in LUAD, based on the StarBase database (Fig. 6C). The results also demonstrated that NETO2 expression was upregulated in LUAD cells (Fig. 6D). Furthermore, NETO2 mRNA and protein expression levels were markedly inhibited following transfection with miR-193a-5p mimics, but promoted following transfection with miR-193a-5p inhibitor in A549 and H1299 cells (Fig. 6E and F). Then, the knockdown of NETO2 in the cells transfected with siRNA was confirmed using RT-qPCR 
(Fig. 6G). Similarly, Transwell, MTT and western blot assays were performed, and the results indicated that NETO2 knockdown distinctly inhibited the invasion, migration (Fig. 6H), proliferation (Fig. 7A) and EMT process (Fig. 7B) in A549 and H1299 cells compared with the NC group. Notably, these effects on the malignant phenotype of LUAD cells were markedly reversed following co-transfection with miR-193a-5p inhibitor or pcDNA-SNHG17 (Fig. 7A and B). Correlation analysis between NETO2-miR-193a-5p and NETO2-SNHG17 was performed and the results indicated that NETO2 expression was negatively associated with miR-193a-5p, while it was positively associated with SNHG17 in cancer tissues (Fig. 7C and D). Notably, NETO2 expression was rescued following transfection with miR-193a-5p inhibitor or pcDNA-SNHG17 (Fig. 7E and F). Taken together, these results suggest that SNHG17 promotes the proliferation, migration, invasion and EMT process of cancer cells by suppressing miR-193a-5p expression to increase NETO2 expression.

\section{Discussion}

Increasing evidence suggest that IncRNAs are aberrantly expressed in different types of human cancer (31). lncRNAs play pivotal roles in cancer, including regulating pathological processes by targeting cell proliferation, migration, invasion and metastasis $(32,33)$. The results of the present study verified that SNHG17 acts as an oncogene in LUAD, whereby SNHG17 expression was significantly upregulated in LUAD cancer tissues and cells. The progression and poor prognosis of patients with LUAD was positively associated with high SNHG17 expression. The present study focused on the association between SNHG17 expression and overall survival time of patients with LUAD. However, there was insufficient data on survival outcome. Thus, further studies are required to perform multivariate analysis, which may be able to determine other prognostic factors associated with LUAD.

Previous studies have reported the carcinogenic role of SNHG17. For example, SNHG17 contributes to the progression of pancreatic cancer by sponging miR-942 (17). Furthermore, SNHG17 was upregulated in HCC, and promoted HCC cell proliferation, migration and invasion by targeting miR-3180-3p and RFX1 expression (34). SNHG17 is also associated with prostate cancer progression, whereby SNHG17 knockdown inhibits cell proliferation, migration, invasion and EMT, and promotes apoptosis by directly targeting miR-339-5p and functions as a ceRNA to upregulate STAT5A expression (35). The results of the present study are consistent with previous findings $(17,34,35)$, confirming that SNHG17 functions as an oncogene, and thus suggesting that SNHG17 may be a potential biomarker for clinical diagnosis of LUAD.

It has been reported that IncRNAs affect cancer progression by regulating the expression of miRNAs (36). The results of the present study demonstrated that SNHG17 functions as a ceRNA by sponging miR-193a-5p and controlling its expression. Notably, miR-193a-5p expression was downregulated in LUAD cancer tissues and cells. miR-193a-5p expression is downregulated in other types of cancer tissues, such as cholangiocarcinoma, LUAD and thymoma. Functionally, overexpression of miR-193a-5p significantly blocked SNHG17 expression, cell proliferation and EMT. Notably, these effects were reversed following miR-193a-5p knockdown. Consistent with the results of the present study, the role of miR-193a-5p as a tumor suppressor has been extensively confirmed $(27,28,37)$. For example, miR-193a-5p inhibits breast cancer progression by targeting SNHG1 and inactivating the expression of the oncogene HOXA1 (37). Overexpression of SPOCK1 contributes to the proliferation and invasion of HCC cells, but as a target of SPOCK1, miR-193a-5p inhibits HCC progression by decreasing SPOCK1 expression (27). Furthermore, as a tumor suppressor in colorectal cancer, the overexpression of miR-193a-5p suppressed the migratory ability of cells (28). Notably, miR-193a-5p expression is significantly upregulated in pancreatic cancer, which is associated with poor prognosis, and overexpression of miR-193a-5p promotes cell metastasis (38). Taken together, these results suggest that miR-193a-5p acts as a tumor suppressor in different types of cancer.

To further investigate the role of miR-193a-5p in inhibiting LUAD progression, bioinformatics analysis and the dual-luciferase report assay were performed, which confirmed that NETO2 is a direct target of miR-193a-5p. Previous studies have reported that NETO2 plays a critical role in cancer progression. For example, NETO2 expression was significantly upregulated in gastric cancer (GC) tissues compared with that in normal tissues, and positively associated with cancer stage and lymph node metastasis (39). Furthermore, overexpression of NETO2 promotes migration, invasion and EMT of GC cells (40). NETO2 is also highly expressed in esophageal cancer, which promotes proliferation and metastasis of cancer cells to accelerate cancer progression by activating the PI3K/AKT and ERK pathway (41). The results of the present study demonstrated that NETO2 expression is upregulated in LUAD tumor tissues and cells, and the methylation level decreased in the promoter region of NETO2. In addition, NETO2 expression was negatively associated with miR-193a-5p expression. Notably, NETO2 knockdown inhibited the proliferation and EMT of LUAD cells, which was markedly reversed following transfection with miR-193a-5p inhibitor or overexpression of SNHG17.

In conclusion, the results of the present study demonstrated that SNHG17 expression is upregulated in LUAD. SNHG17 knockdown inhibited cell proliferation, migration, invasion and EMT, suggesting that SNHG17 contributes to the progression of LUAD by acting as an oncogene. The regulation of SNHG17 in LUAD was realized by targeting the miR-193a-5p/NETO2 axis. Thus, SNHG17 may be used as a novel diagnostic marker or therapeutic target of LUAD. However, further studies are required to determine how the SNHG17/miR-193a-5p/NETO2 axis regulates LUAD progression. Thus, prospective studies will focus on investigating the molecular mechanism and effects of SNHG17 in LUAD tumor growth and tumor immune microenvironment in vivo.

\section{Acknowledgements}

Not applicable.

\section{Funding}

No funding was received. 


\section{Availability of data and materials}

The datasets used and/or analyzed during the present study are available from the corresponding author upon reasonable request.

\section{Authors' contributions}

ZWZ analyzed the patient data. ZWZ and CLW designed the experiments and confirmed the authenticity of all the raw data. ZWZ, YLY, BZ, YCM and CC performed the experiments and analyzed the data. ZWZ and CLW drafted the original manuscript. All authors have read and approved the final manuscript.

\section{Ethics approval and consent to participate}

The present study was approved by the Ethics Committees of Tianjin Medical University Cancer Institute and Hospital (Tianjin, China; approval no. TJWZXYXEC-). Written informed consent was provided by all patients prior to the study start.

\section{Patient consent for publication}

Not applicable.

\section{Competing interests}

The authors declare that they have no competing interests.

\section{References}

1. Nasim F, Sabath BF and Eapen GA: Lung cancer. Med Clin North Am 103: 463-473, 2019.

2. Toumazis I, Bastani M, Han SS and Plevritis SK: Risk-based lung cancer screening: A systematic review. Lung Cancer 147: 154-186, 2020.

3. Howlader N, Forjaz G, Mooradian MJ, Meza R, Kong CY, Cronin KA, Mariotto AB, Lowy DR and Feuer EJ: The effect of advances in lung-cancer treatment on population mortality. N Engl J Med 383: 640-649, 2020.

4. Yang F, Yi F, Zheng Z, Ling Z, Ding J, Guo J, Mao W, Wang X, Wang X, Ding X, et al: Characterization of a carcinogenesisassociated long non-coding RNA. RNA Biol 9: 110-116, 2012.

5. Gelatti ACZ,Drilon A and Santini FC: Optimizing the sequencing of tyrosine kinase inhibitors (TKIs) in epidermal growth factor receptor (EGFR) mutation-positive non-small cell lung cancer (NSCLC). Lung Cancer 137: 113-122, 2019.

6. Schmitz SU, Grote P and Herrmann BG: Mechanisms of long noncoding RNA function in development and disease. Cell Mol Life Sci 73: 2491-2509, 2016.

7. Chen W, Zheng R, Baade PD, Zhang S, Zeng H, Bray F, Jemal A, Yu XQ and He J: Cancer statistics in China, 2015. CA Cancer J Clin 66: 115-132, 2016.

8. Lin JJ, Cardarella S, Lydon CA, Dahlberg SE, Jackman DM, Jänne PA and Johnson BE: Five-year survival in EGFR-mutant metastatic lung adenocarcinoma treated with EGFR-TKIs. J Thorac Oncol 11: 556-565, 2016.

9. Bhan A, Soleimani M and Mandal SS: Long noncoding RNA and cancer: A new paradigm. Cancer Res 77: 3965-3981, 2017.

10. Wahlestedt C: Targeting long non-coding RNA to therapeutically upregulate gene expression. Nat Rev Drug Discov 12: 433-446, 2013.

11. Flippot R, Beinse G, Boileve A, Vibert J and Malouf GG: Long non-coding RNAs in genitourinary malignancies: A whole new world. Nat Rev Urol 16: 484-504, 2019.

12. Sanchez Calle A, Kawamura Y, Yamamoto Y, Takeshita F and Ochiya T: Emerging roles of long non-coding RNA in cancer. Cancer Sci 109: 2093-2100, 2018.
13. Kong X, Duan Y, Sang Y, Li Y, Zhang H, Liang Y, Liu Y, Zhang $\mathrm{N}$ and Yang Q: LncRNA-CDC6 promotes breast cancer progression and function as ceRNA to target CDC6 by sponging microRNA-215. J Cell Physiol 234: 9105-9117, 2019.

14. Ni W, Zhang Y, Zhan Z, Ye F, Liang Y, Huang J, Chen K, Chen L and Ding Y: A novel lncRNA uc.134 represses hepatocellular carcinoma progression by inhibiting CUL4A-mediated ubiquitination of LATS1. J Hematol Oncol 10: 91, 2017.

15. Tao Y, Tang Y, Yang Z, Wu F, Wang L, Yang L, Lei L, Jing Y, Jiang $\mathrm{X}$, Jin $\mathrm{H}$, et al: Exploration of serum exosomal LncRNA TBILA and AGAP2-AS1 as promising biomarkers for diagnosis of non-small cell lung cancer. Int J Biol Sci 16: 471-482, 2020.

16. Liu Y, Li Q, Tang D, Li M, Zhao P, Yang W, Shu L, Wang J, He Z, Li Y and Wang F: SNHG17 promotes the proliferation and migration of colorectal adenocarcinoma cells by modulating CXCL12-mediated angiogenesis. Cancer Cell Int 20: 566, 2020.

17. Zhao L, Ye J, Lu Y, Sun C and Deng X: lncRNA SNHG17 promotes pancreatic carcinoma progression via cross-talking with miR-942. Am J Transl Res 13: 1037-1050, 2021.

18. Du Y, Wei N, Hong J and Pan W: Long non-coding RNASNHG17 promotes the progression of breast cancer by sponging miR-124-3p. Cancer Cell Int 20: 40, 2020.

19. Jia Y, Duan Y, Liu T, Wang X, Lv W, Wang M, Wang J and Liu L: LncRNA TTN-AS1 promotes migration, invasion, and epithelial mesenchymal transition of lung adenocarcinoma via sponging miR-142-5p to regulate CDK5. Cell Death Dis 10: 573, 2019.

20. Li C, Zhao W, Pan X, Li X, Yan F, Liu S, Feng J and Lu J: LncRNA KTN1-AS1 promotes the progression of non-small cell lung cancer via sponging of miR-130a-5p and activation of PDPK1. Oncogene 39: 6157-6171, 2020.

21. Hua Q, Jin M, Mi B, Xu F, Li T, Zhao L, Liu J and Huang G: LINC01123, a c-Myc-activated long non-coding RNA, promotes proliferation and aerobic glycolysis of non-small cell lung cancer through miR-199a-5p/c-Myc axis. J Hematol Oncol 12: 91, 2019.

22. Livak KJ and Schmittgen TD: Analysis of relative gene expression data using real-time quantitative PCR and the 2(-Delta Delta C(T)) method. Methods 25: 402-408, 2001.

23. Yang S, Liu Y, Li MY, Ng CSH, Yang SL, Wang S, Zou C, Dong Y, Du J, Long X, et al: FOXP3 promotes tumor growth and metastasis by activating $\mathrm{Wnt} / \beta$-catenin signaling pathway and EMT in non-small cell lung cancer. Mol Cancer 16: 124, 2017.

24. Tang Q, Chen J, Di Z, Yuan W, Zhou Z, Liu Z, Han S, Liu Y, Ying G, Shu X and Di M: TM4SF1 promotes EMT and cancer stemness via the $\mathrm{Wnt} / \beta$-catenin/SOX2 pathway in colorectal cancer. J Exp Clin Cancer Res 39: 232, 2020.

25. Pan J, Fang S, Tian H, Zhou C, Zhao X, Tian H, He J, Shen W, Meng X, Jin X and Gong Z: lncRNA JPX/miR-33a-5p/Twist1 axis regulates tumorigenesis and metastasis of lung cancer by activating Wnt/ $\beta$-catenin signaling. Mol Cancer 19: 9, 2020.

26. Luo H, Xu C, Le W, Ge B and Wang T: IncRNA CASC11 promotes cancer cell proliferation in bladder cancer through miRNA-150. J Cell Biochem 120: 13487-13493, 2019.

27. Li P, Xiao Z, Luo J, Zhang Y and Lin L: MiR-139-5p, miR-940 and miR-193a-5p inhibit the growth of hepatocellular carcinoma by targeting SPOCK1. J Cell Mol Med 23: 2475-2488, 2019.

28. Shirafkan N, Shomali N, Kazemi T, Shanehbandi D, Ghasabi M, Baghbani E, Ganji M, Khaze V, Mansoori B and Baradaran B: microRNA-193a-5p inhibits migration of human HT-29 colon cancer cells via suppression of metastasis pathway. J Cell Biochem: Dec 2, 2018 (Epub ahead of print).

29. Chen J, Gao S, Wang C, Wang Z, Zhang H, Huang K, Zhou B, Li H, Yu Z, Wu J and Chen C: Pathologically decreased expression of miR-193a contributes to metastasis by targeting WT1-E-cadherin axis in non-small cell lung cancers. J Exp Clin Cancer Res 35: 173, 2016.

30. Donehower LA, Soussi T, Korkut A, Liu Y, Schultz A, Cardenas M, Li X, Babur O, Hsu TK, Lichtarge O, et al: Integrated analysis of TP53 gene and pathway alterations in the cancer genome atlas. Cell Rep 28: 1370-1384.e5, 2019.

31. Sun Z, Yang S, Zhou Q, Wang G, Song J, Li Z, Zhang Z, Xu J, Xia K, Chang Y, et al: Emerging role of exosome-derived long non-coding RNAs in tumor microenvironment. Mol Cancer 17: 82, 2018.

32. Hui B, Ji H, Xu Y, Wang J, Ma Z, Zhang C, Wang K and Zhou Y: RREB1-induced upregulation of the lncRNA AGAP2-AS1 regulates the proliferation and migration of pancreatic cancer partly through suppressing ANKRD1 and ANGPTL4. Cell Death Dis 10: 207, 2019.

33. Li Y, Zhang X, Zheng Q, Zhang Y, Ma Y, Zhu C, Yang L, Peng X, Wang Q, Wang B, et al: YAP1 inhibition in HUVECs is associated with released exosomes and increased hepatocarcinoma invasion and metastasis. Mol Ther Nucleic Acids 21: 86-97, 2020. 
34. Ma T, Zhou X, Wei H, Yan S, Hui Y, Liu Y, Guo H, Li Q, Li J, Chang Z and Mu XX: Long non-coding RNA SNHG17 upregulates RFX1 by sponging miR-3180-3p and promotes cellular function in hepatocellular carcinoma. Front Genet 11: 607636, 2020.

35. Wu G, Hao C, Qi X, Nie J, Zhou W, Huang J and He Q: LncRNA SNHG17 aggravated prostate cancer progression through regulating its homolog SNORA71B via a positive feedback loop. Cell Death Dis 11: 393, 2020.

36. Chan JJ and Tay Y: Noncoding RNA: RNA regulatory networks in cancer. Int J Mol Sci 19: 1310, 2018.

37. Li J, Zeng T, Li W, Wu H, Sun C, Yang F, Yang M, Fu Z and Yin Y: Long non-coding RNA SNHG1 activates HOXA1 expression via sponging miR-193a-5p in breast cancer progression. Aging (Albany NY) 12: 10223-10234, 2020.

38. Li M, Wu P, Yang Z, Deng S, Ni L, Zhang Y, Jin L and Pan Y: miR-193a-5p promotes pancreatic cancer cell metastasis through SRSF6-mediated alternative splicing of OGDHL and ECM1. Am J Cancer Res 10: 38-59, 2020.

39. Liu JY, Jiang L, He T, Liu JJ, Fan JY, Xu XH, Tang B, Shi Y, Zhao YL, Qian F, et al: NETO2 promotes invasion and metastasis of gastric cancer cells via activation of PI3K/Akt/NF- $\kappa \mathrm{B} / \mathrm{Snail}$ axis and predicts outcome of the patients. Cell Death Dis 10: 162, 2019.
40. Han Y, Wu N, Jiang M, Chu Y, Wang Z, Liu H, Cao J, Liu H, $\mathrm{Xu} \mathrm{B}$ and Xie X: Long non-coding RNA MYOSLID functions as a competing endogenous RNA to regulate MCL-1 expression by sponging miR-29c-3p in gastric cancer. Cell Prolif 52: e12678, 2019.

41. Xu JC, Chen TY, Liao LT, Chen T, Li QL, Xu JX, Hu JW, Zhou PH and Zhang YQ: NETO2 promotes esophageal cancer progression by inducing proliferation and metastasis via PI3K/AKT and ERK pathway. Int J Biol Sci 17: 259-270, 2021.

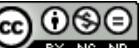

This work is licensed under a Creative Commons Attribution-NonCommercial-NoDerivatives 4.0 International (CC BY-NC-ND 4.0) License. 